\title{
Das lange erwartete etymologische Wörterbuch des Mari
}

GÁBor BereczKI: Etymologisches Gábor Bereczki, der sein LebensWörterbuch des Tscheremissi- werk im Bereich des Mari (Tscheschen (Mari): Der einheimische remissischen) leistete, widmete Wortschatz. Nach dem Tode des sich nach seiner Pensionierung der Verfassers herausgegeben von Arbeit an einem etymologischen Klára Agyagási und Eberhard Wörterbuch des Mari. Das postWinkler. Veröffentlichungen der hum erschienene Wörterbuch ist Societas Uralo-Altaica 86. Wies- das letzte wissenschaftliche Erbe, baden: Harrassowitz Verlag, das der 2012 verstorbene Bereczki 2013. $332 \mathrm{~S}$.

der wissenschaftlichen Gemein- 
schaft hinterließ. Seine ersten Beiträge zur Etymologie des Mari erschienen bereits in den 1950er Jahren, und in seinen Untersuchungen aus verschiedenen Jahrzehnten präsentierte er zahlreiche neue Erkenntnisse u.a. über den Einfluss der türkischen Sprachen, insbesondere des Tschuwaschischen, auf das Mari. Auch die Beobachtung der Kontakte zwischen den permischen Sprachen, vor allem zwischen dem Udmurtischen und dem Mari eröffnete eine wichtige neue Perspektive auf die marische Sprachgeschichte.

Ungeachtet seines Obertitels bietet das Buch keine erschöpfende Synthese des etymologischen Hintergrundes des marischen Wortschatzes; vielmehr hat der Untertitel Der einheimische Wortschatz eine erhebliche Bedeutung für den Inhalt. Es handelt sich folglich um eine wesentlich erweiterte und überarbeitete Fortsetzung des etymologischen Verzeichnisses in Bereczkis zweibändigem Werk Grundzüge der tscheremissischen Sprachgeschichte I-II (Bereczki 1992-1994).

Das Wesen des posthum veröffentlichten und bei der Herausgabe in den Kommentarteilen ergänzten etymologischen Wörterbuches tritt bereits im Vorwort zu Tage. Gábor Bereczki arbeitete jahrelang mit der Turkologin Klára Agyagási zusammen, die das Buch gemeinsam mit Eberhard Winkler druckfertig gemacht hat. Das Grundkorpus der etymologischen Untersuchung des Mari bildeten ursprünglich etwa 5000 Wörter, von denen rund 2500 tschuwaschischer oder tatarischer Herkunft sind und nur etwa 500 zum alten finnisch-ugrischen Wortschatz zählen oder diesem früher zugeordnet wurden. Zum Wortschatz finnisch-ugrischer Herkunft werden im vorliegenden Werk auch die aus den permischen Sprachen ins Mari gelangten Wörter gezählt, eines der wichtigsten Ergebnisse von Bereczkis Lebenswerk. Viele wertvolle Beobachtungen auch in dem nun erschienenen Buch betreffen gerade den über das Permische ins Mari gelangten Wortschatz. Dazu zählen z. B. ća $a k$,voll, dichtgedrängt', kaške ,Schimmel', kaškem ,schimmelig werden, veschimmeln', lüs

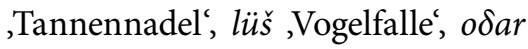
,Spross, Trieb', ter-, tor- usw. , bei, zu, nach; mit', tušto ,Rätselfrage' und mehrere andere. Dagegen handelt es sich zum Beispiel bei šürem,(ein) schmieren' und seinen vermutlichen permischen Entsprechungen um voneinander unabhängige türkische Lehnwörter.

Entgegen seinem Obertitel deckt das hier zu besprechende Wörterbuch nur einen kleinen Teil des marischen Wortschatzes ab. Auch aus dem Grundwortschatz bleibt vieles unberücksichtigt, da 
der Einfluss der türkischen Sprachen so stark ist. Am zweiten, die wolgabulgarischen, tschuwaschischen und russischen Lehnwörter im Mari behandelnden Teil arbeitet Agyagási weiterhin; er wird hoffentlich bald erscheinen und den vorliegenden Band ergänzen.

\section{Technische Lösungen}

Für die Lemmata wurde die in der Praxis der wiesenmarischen Schriftsprache entsprechende Variante gewählt, die in lateinischer Schrift transkribiert wurde, gemäß der dem Mari angepassten finnisch-ugrischen Transkription. Die alphabetische Ordnung beruht auf dem angewandten lateinischen Modell, aber unmittelbar nach dem Hauptlemma wird auch die Form angegeben, die der Orthografie der beiden marischen Schriftsprachen, Wiesen- und Bergmari, entspricht. Die Entsprechungen in den verwandten Sprachen wurden aufgrund der Angaben in dem als Hauptquelle verwendeten Uralischen etymologischen Wörterbuch (1988, im Folgenden UEW) zusammengestellt, und auch die Rekonstruktion des gemeinsamen Wortschatzes der uralischen Sprachen wurde dem UEW entnommen. Das Buch enthält insgesamt 585 Lemmata, davon sind etwa 100 Lehnwörter oder Ableitungen.
Während der Abfassung des Wörterbuchs (2008) erschien das an der Universität Turku erstellte, von der Finnisch-Ugrischen Gesellschaft herausgegebene Tscheremissische Wörterbuch (TschWb), dessen Erklärungen deutschsprachig sind. Im vorliegenden etymologischen Wörterbuch wird in Einzelfällen auch darauf verwiesen, doch wurden beispielsweise die Wortbedeutungen unabhängig verfasst. Dies wird vor allem bei polysemen Wörtern deutlich.

Eine der problematischsten Grundentscheidungen ist die Verwendung des 1988 erschienenen UEW als Hauptquelle für die Rekonstruktion der ursprachlichen Ebene der anderen uralischen Sprachen und Wörter, mit der die vorliegende Monografie einen Dialog führt. Ein entsprechender Dialog mit anderen Untersuchungen fehlt. So wird das vorliegende Buch an die Forschungssituation der 1970er und 1980er Jahre gebunden. Grundlage dieser Entscheidung war praktisches Denken: Es ist effektiver, das Material aus dem UEW zusammenzustellen, als zu versuchen, zahlreiche Primärquellen in verschiedenen Sprachen zu benutzen. Allerdings konstatiert auch Bereczki in der von ihm selbst verfassten Einleitung, dass sich der Forschungsstand verändert hat: „Seit dem Erscheinen des [UEW] 
ist im Laufe des vergangenen Vierteljahrhunderts eine Unzahl an Wörterbüchern erschienen." Er fügt hinzu, dass er diese Wörterbücher auch bei der Überprüfung der Etymologie mehrerer marischer Wörter genutzt habe.

Die Nachteile der Anlehnung an das UEW werden weniger im Wortschatzmaterial sichtbar als vielmehr in der eigentlichen etymologischen Analyse und in den Rekonstruktionen, die das Fundament der sprachgeschichtlichen Interpretationen bilden. Leider wurde das UEW bei seinem Erscheinen nicht kritisch beurteilt. Wichtig für die Forschungskonstellation ist, dass zum Beispiel die auf einzelne uralische Sprachen gerichtete Perspektive und diejenige der Lehnwortforschung $\mathrm{zu}$ einer Gesamtheit verbunden werden. Die größte Schwäche des UEW ist die Unsicherheit der Rekonstruktion, insbesondere hinsichtlich der Vokalgeschichte. Es nahm nicht Stellung zu der von Juha Janhunen 1981 in SUSA vorgeschlagenen Vokalrekonstruktion der uralischen Sprachen (Janhunen 1981). Im selben Jahr wie das UEW erschienen der Beitrag von Mikko Korhonen über die Phonotaktik des Vokalismus der nichtersten Silben (Korhonen 1988) und der Beitrag von Pekka Sammallahti, der die Gesamtent- wicklung des Vokalismus behandelte (Sammallahti 1988), wodurch ein großer Teil des auch im UEW behandelten Materials in ein neues Licht gerückt wurde. Seither sind neue Untersuchungen erschienen, so etwa der an Janhunen und Sammallahti anknüpfende Beitrag von Ante Aikio (Aikio 2012). Aus zeitlichen Gründen wäre es natürlich unangemessen, zu erwarten, dass der letztgenannte Beitrag im etymologischen Wörterbuch des Mari berücksichtigt worden wäre, doch insgesamt haben die Neueinschätzung der Vokalgeschichte und die Fortschritte der Lehnwortforschung im Bereich der alten uralisch-indogermanischen Sprachkontakte eine wesentliche Veränderung der Forschungssituation herbeigeführt.

Der Vokalismus, der in den früheren Modellen unlogisch erschien und sporadische Lautentwicklungen voraussetzte, findet in den genannten Beiträgen eine haltbare Erklärung, allerdings größtenteils erst zu einem Zeitpunkt, als das UEW bereits druckfertig war. Zuzugeben ist auch, dass zum Beispiel in Sammallahtis Beitrag der schwächste Punkt vielleicht gerade die Behandlung des marischen Materials ist, weshalb er nicht unmittelbar auf die Untersuchung der marischen Wörter angewandt werden kann. 
Der etymologische Hintergrund des Wortschatzes des Mari

Von entscheidender Wichtigkeit hinsichtlich der Herkunft des marischen Wortschatzes dürfte die Unterscheidung der türkischen, d.h. der bulgarischen, tschuwaschischen und tatarischen Schicht sein. Dies ist das Spezialgebiet von Klára Agyagási, die das posthume Werk redigiert hat. Aus der Sicht des Lesers ist es jedoch bedauerlich, dass das etymologische Wörterbuch des Mari nun, da es endlich erscheint, wegen der vorgenommenen Begrenzung zwangsläufig unvollständig ist. Im Fokus des hier zu besprechenden Werkes steht also der auf das Uralische zurückgehende Wortschatz des Mari; hinzu kommen auch einige alte indogermanische Entlehnungen, die freilich nicht systematisch gesammelt wurden. Das Grundkonzept erinnert an das in Ungarn (1967-78) erschienene Werk Magyar szókészlet finnugor elemei (MSzFE). Wie bereits erwähnt, misst es sich auch mit der 20 Jahre zuvor erschienenen Geschichte der marischen Sprache von Bereczki, gegenüber der das nun vorliegende Werk wesentlich umfangreicher ist. Der Behandlung des Materials und den Begründungen wird erheblich mehr Platz eingeräumt, und den wissenschaftlichen Gepflogenheiten entspre- chend werden auch Etymologien einbezogen, die Bereczki als falsch erklärt. Besonderes Gewicht legt er auf die Kommentierung der lautlichen Entwicklung.

Bereits die ersten Lemmata veranschaulichen die Struktur der Untersuchung. Das UEW stellt wiesenmari $a \eta$, bergmari $\ddot{a} \eta$,Öffnung, Spalt; Eingang; Mündung; Ende oder Anfang' (S. 1) unter Vorbehalt neben Worte aus dem Saamischen, dem Mordwinischen, den permischen und ugrischen Sprachen. Dass das Wort im Mari mit den Worten der anderen uralischen Sprachen in Verbindung zu bringen sei, ist Bereczki zufolge schon wegen der Vokalvertretung $a \sim \ddot{a}$ fraglich, da im marischen Wortschatz uralischer Herkunft keine entsprechenden Fälle begegnen. Nach sorgfältiger Argumentation wird festgestellt, dass das Wort mit tschuwaschisch ana ,отверстие, проем' zu verbinden ist.

\section{Berichtigungen zu den Etymologien des UEW}

Die Strategie bei der Abfassung des vorliegenden Buches ist es, die Behauptungen des UEW zu kommentieren. Mehrfach weist Bereczkis etymologisches Wörterbuch nach, dass die im UEW angeführte Etymologie eines marischen Wortes falsch ist. Beispielsweise ist elte, 
eltem ,Schoß, Bündel' ein tschuwaschisches Lehnwort, wie bereits Martti Räsänen 1920 postulierte. Um tschuwaschische Lehnwörter, nicht um wahrscheinliche oder mögliche Entsprechungen zu Wörtern der anderen finnischugrischen Sprachen handelt es sich auch bei enam, ohne Flamme brennen, glühen, schwelen'.

Auch bei mehreren anderen Wörtern weist Bereczki nach, dass die im UEW vorgebrachte Analyse falsch ist. Das Wort kaške ,Schimmel', kaškem ,schimmelig werden, verschimmeln' wurde ungenau aus Ramstedts Wörterbuch zitiert und ist nach Bereczkis Ansicht höchstwahrscheinlich eine permische Entlehnung. Mari loso ,Einkerbung, Markierung, Radspur etc.,'

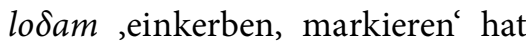
keine Verbindung zu dem finnischen Wort latu ,Spur, Skispur, die auch SSA für möglich hält, sondern es könnte sich Bereczki zufolge um ein iranisches Lehnwort handeln. Allerdings wird die Entlehnungsquelle nicht näher genannt. Das Wort lop ,Niederung' ist nicht aus dem ururalischen *lampi ,Pfütze, Teich, Moor abzuleiten, sondern eher aus der Form ${ }^{\star} l a p p a \sim{ }^{*} l a p$ pi. Mari omaš ,Hütte, Zelt' lässt sich problemlos als Entsprechung des finnischen Wortes uudin, Vorhang, Gardine' und der mit ihm verbundenen ugrischen Wörter betrachten, obwohl UEW und SSA es nicht anführen. Die Rekonstruktion lässt in Übereinstimmung mit der Linie des UEW den Vokalismus der nichtersten Silben offen: ${ }^{*} u \delta \varepsilon-m \varepsilon$,Schlafzelt, Mückenzelt'. Auch ońâš ,weiter' und die von Bereczki damit verbundenen umbal ,entfernt' und una ,sieh, da' fehlen in der Liste des UEW, obwohl sie dasselbe pronominale Element enthalten wie zum Beispiel mordw. E ombo : omboće, $\mathrm{M}$ omba : omboćä ,anderer, zweiter, udm. otin ,dort', ko. ata ,hier', ung. $a z, a$,jener, der etc. Das hierzu gehörende deiktische Proadverb ott , dort' erwähnt Bereczki aus irgendeinem Grund nicht, obwohl die etymologische Beziehung zum Beispiel im etymologischen Wörterbuch des Ungarischen konstatiert wird (EWU 1075-1076). Mari šuršo ,Floh' kann auch aus lautlichen Gründen nicht fi. sonsar ,Floh', mordw. E čičav und den damit verbundenen ugrischen Wörtern entsprechen und ist eher eine Ableitung aus dem Verb šurem ,stechen'. Die Wörter šârt ,böser Geist, Teufel' und waja ,Besatz, Saumnaht, Verbrämung' sind türkische Lehnwörter, im UEW ungenau dargestellt. Der Gleichsetzung des im Ostseefinnischen fi. kerta ,Mal, Schicht', Saamischen und Mordwinischen begegnenden baltischen Lehnworts mit der zweiten Komponente des marischen 
Kompositums šukerte, schon lange, schon längst', die in der etymologischen Literatur wiederholt erwähnt wird, steht Bereczki äußerst kritisch gegenüber. Dafür gibt es einen gewichtigen Grund, der in der Literatur allerdings nicht erwähnt wird. Mari šukerte wurde in den Analysen falsch segmentiert, obwohl die Zweimorphemigkeit bei synchronischer Betrachtung transparent ist: šuko, viel' + ertam ,gehen, kommen, wandern usw. ' dies erwähnt freilich auch das vorliegende Werk nicht.

Bereczki korrigiert auch zahlreiche andere Gleichsetzungen des UEW, wie z. B. kajem, gehen, reisen', kinde ,Brot; Getreide', für das das UEW eine auch lautlich ungeeignete zufällige Entsprechung aus Agricolas Götterverzeichnis anführt, luk ,Ecke, Hausecke, Biegung', maća ,Netzsack in der Mitte des Fischernetzes', malāš ,Holzdeckel der Öffnung eines Bienenstockes in einem Baumstumpf ${ }^{c}, \check{s} u$ ,Gesundheit, gesund', taće ,heute, tić ,voll', ueštam gähnen', tušto ,Rätselfrage $\sim$ tuštem, eine Rätselfrage stellen', üpš ,Geruch ‘ $\sim$ üpšem ,einen Geruch haben, stinken'.

Die Neueinschätzung der einzelnen Etymologien ist konsequent. Präzisierungen können bei lautlich ungenauen oder fehlerhaften Analysen erforderlich sein. Bei mehreren Wörtern, die im Kontext des uralischen Wortschatzes behandelt werden, handelt es sich tatsächlich um tschuwaschische oder permische Lehnwörter. Gelegentlich zeigt sich auch, dass das marische Wort eher zu einer anderen Wortfamilie gehört. Wiederholt erweist sich zudem, dass es für die mit dem Mari verglichenen ostseefinnischen Wörter ein plausibleres Lehnoriginal gibt. In dieser Hinsicht stützt sich das Wörterbuch auf das Werk Suomen sanojen alkuperä [Die Herkunft der Wörter des Finnischen] (SSA). Im UEW ist diese Perspektive durchgängig sehr schwach vertreten, und auch seit dem Erscheinen des SSA hat die Forschung erhebliche Fortschritte gemacht.

\section{Problematische Interpretationen}

Es gibt vieles zu korrigieren und $\mathrm{zu}$ präzisieren, weshalb der Leser daran zu zweifeln beginnt, ob die strukturelle Lösung, die Lemmata in der unmittelbar dem UEW entnommenen Form zu präsentieren, gelungen ist. Bei dieser Ausgangskonstellation tritt nicht das Thema des Buches der Wortschatz finnisch-ugrischer Herkunft im Mari - in den Vordergrund, sondern die Art der Behandlung des Themas im UEW.

Bei mehreren Etymologien ist es angebracht, sie aus einem anderen Blickwinkel zu betrachten und alternative Erklärungen zu erörtern. 
Mari koćo ,bitter' (S. 66-67) wird in der etymologischen Literatur dem finnischen Wort katkera ,bitter, verbittert ${ }^{\star}$ zur Seite gestellt, das Entsprechungen im Karelischen und Ingrischen hat. Das SSA postuliert hierfür keine Entsprechungen in den anderen uralischen Sprachen außer dem Mari. Das UEW (113) und Bereczki in Anlehnung daran schlagen Entsprechungen im Saamischen, Udmurtischen und in den ugrischen Sprachen vor, die SSA lautlich nicht für möglich hält. Da das nur im nördlichen Ostseefinnischen begegnende katkera eine Ableitung ist, wäre es begründet, bei dem marischen Wort $\mathrm{zu}$ überlegen, ob es nicht doch $\mathrm{zu}$ demselben Stamm gehört, den finnisch katku ,stechender Gestank von Angebranntem od. Rauch', wepsisch kačk ,Rauch etc.', mordwinisch E kačamo, Rauch' < ${ }^{\star} k a c ̌ a$ repräsentieren.

Olli Nuutinen schlug bereits 1989 für das Wort SaaN jávri, fi. järvi, MdE eŕke, Ma. jer,See' (S. 29) eine beachtenswerte baltische Etymologie vor, die im UEW nicht kommentiert und im SSA nur im Kommentar zur Bibliografie erwähnt wird; auch in dem hier zu besprechenden Buch wird diese alternative Erklärung nicht näher kommentiert.

Das Verb jükšem ,kalt werden (S. 39) und das Adjektiv jüštö ,kalt
(S. 40) werden als separate Lemmata ohne Querverweis angeführt, obwohl sie aus rein marischer Perspektive ganz offensichtlich etymologisch von gleicher Herkunft sind. Die als Quellen verwendeten Werke SSA und UEW nehmen an, dass es sich so verhält, doch wegen der in den westlichen uralischen Sprachen zu beobachtenden Doppelrepräsentation müssen zwei parallele Stämme *jakša ja *jäkšä rekonstruiert werden.

Für die marischen Wörter kârem ,schlagen, hauen; dreschen ${ }^{c}$ (S. 6o), purem, hineingehen, eintreten, eingehen' (S. 202) und ihre mordwinischen und permischen Entsprechungen habe ich eine indoiranische Lehnetymologie vorgeschlagen (Grünthal 2001, 2002), zu der im vorliegenden Buch nicht Stellung genommen wird. Dagegen werden hier und an zahlreichen anderen Stellen als Lehnübersetzungen Konverbkonstruktionen angeführt, die eine Parallele im Tschuwaschischen haben. Die indogermanische Herkunft wird auch bei dem seit mehr als hundert Jahren bekannten Lehnwort mari šur ,Horn; Schopf (am Kopf eines Vogels)' (S. 257) und seinen etymologischen Entsprechungen fi. sarvi ,Horn, Waldhorn', ung. szarv ,Horn, Gehörn' usw. nicht erwähnt. Dagegen wird die indoiranische Herkunft für das Wort šü $\delta \ddot{o}, 100^{\circ}$ 
(S. 262) und das weniger verbreitete urdem , halten (Tiere), auf-, großziehen (Kinder)' (S. 297) festgestellt. Für das letztere sind Entsprechungen in den permischen Sprachen bekannt.

Bei dem Verb kojam, sichtbar sein, erscheinen' (S. 68) folgt das Werk der Linie des UEW und lässt die problemlos als Entsprechung in Frage kommende, auch im SSA angeführte ostseefinnische Wortfamilie, fi. kajastaa ,schimmern, dämmern, hell werden etc.' unerwähnt.

Das als einzige ostseefinnische Entsprechung des Wortes kowâ ,Welle (S. 78) erwähnte wepsische kobe : kopken ,Schaum' gehört nicht $\mathrm{zu}$ den genannten marischen und permischen Wörtern, sondern seine Entsprechungen sind saamisch guohpa : guohppaga ,Schimmel', südestnisch kope : koppe,Muffigkeit, Schimmel', ein von Koivulehto (1999 (1992): 323) vorgeschlagenes germanisches Lehnwort. Allerdings erwähnt auch Koivulehto das wepsische Wort in diesem $\mathrm{Zu}$ sammenhang nicht. Die Lehnetymologie schließt die Möglichkeit aus, dass das marische Verb kupem ,schimmeln' mit dem erwähnten Substantiv im Saamischen zu verbinden wäre (S. 90).

Aufgrund dessen, was hinsichtlich der Entwicklung der finnischugrischen Sprachen in der Bronze- und Eisenzeit angenommen wird, kann die Metallbezeichnung kürtńö ,Eisen' (S. 102) nicht auf die gemeinsame Ursprache des Mordwinischen, des Mari und des Permischen zurückgeführt werden. Die iranische Herkunft der Wörter mit der Bedeutung Eisen hat bereits Joki angenommen (1973: 273), was in keiner Weise kommentiert wird.

Bereczki weist nach, dass das Wort omaš ,Hütte, Zelt' (S. 172) in denselben Kontext gehört wie fi. uudin ,Vorhang, Gardine', udm. in ,Bettvorhang', komi von, en id., chant. olaw ,Schlafzelt' usw., obgleich zum Beispiel SSA und UEW das marische Wort nicht erwähnen. Das vorliegende Buch beschränkt sich darauf, die Rekonstruktion ${ }^{*} u \delta 3-m 3$ anzuführen und für das Mari eine gleichartige Entwicklung festzustellen wie bei dem Wort omo ,Schlaf, Traum ' $<{ }^{*} o \delta a-m 3$, das etymologische Entsprechungen im Saamischen, Mordwinischen und Mansischen hat. Die Verbreitungsgebiete beider Etymologien decken sich vollständig, was Anlass zu weitergehenden Analysen geben sollte.

Das Verb pörtam, sich drehen, rotieren' im Mari (S. 197), bei dem es sich nebenbei bemerkt um eine offensichtliche Kausativableitung handelt, steht unverkennbar in Verbindung mit den ostseefinnischen Wörtern mit der Bedeutung ,Rad' und ,Drehen', vgl. fi. pyörä ,Rad', pyöriä ,sich drehen, sich winden', 
pyörittää ,drehen', auch wenn Bereczkis Quellen SSA und UEW diese nicht erwähnen. Der Kommentar des SSA über die Deskriptivität des Wortes beispielsweise ist ein für dieses Werk leider typisches, schlecht begründetes Erklärungsmotiv.

Bei dem Wort šänga ,Reisig (S. 222) beschränkt sich das hier zu besprechende Buch auf den im UEW vorgenommenen Vergleich mit dem finnischen Wort sänki ,Stoppeln', kommentiert dagegen nicht die im SSA erwähnten Entsprechungen im Saamischen, Mordwinischen, den permischen und ugrischen Sprachen - SSA wiederum erwähnt das Mari nicht.

Saamisch suovdi, Kieme, Rachen' (S. 238) ist, im Gegensatz zu dem, was auch das UEW postuliert, nicht die etymologische Entsprechung des marischen Wortes šoঠo ,Lunge‘. Vielmehr gehört es zu einem im Ostseefinnischen begegnenden Wort, vgl. fi. hauta ,Graben, Grube etc.' und ist eine sehr frühe germanische Entlehnung (LÄGLOS I (1991): 89-90).

Bei dem Wort šoy ,Schaum (S. 244) wird darauf hingewiesen, dass das ostseefinnische, vgl. fi. hii$v a$,Hefe ${ }^{\varsigma}$ vom Vokalismus her nicht recht mit ihm in Verbindung $\mathrm{zu}$ bringen ist. In diesem Zusammenhang wäre es angebracht gewesen, dazu Stellung zu nehmen, dass das ostseefinnische Wort, wie auch SSA anführt, vermutlich ein baltisches Lehnwort ist, weshalb die Zusammenstellung mit dem Mari nicht korrekt ist.

Baltischer Herkunft ist auch das ostseefinnische Wort fi. hara, Egge; Draggen' < *̌šra < PB *̌̃arā, vgl. lett. zars ,Zweig', lit. žarà, eine Feststellung, die Jorma Koivulehto auch dem Rezensenten gegenüber mündlich geäußert hat und die Aikio (2009: 149) zitiert. Mari šor-wondo ,Rechen' (S. 247) hat trotz der lautlichen und semantischen Ähnlichkeit keine Verbindung zu diesem Wort, sondern ist ein transparentes Kompositum šor ,Morast, Dreck, Schmutz etc.' + wondo ,Stiel', dessen Komponenten gemeinsam ein Wort mit der gleichen Bedeutung wie finnisch harava bilden. Auch wegen der sehr begrenzten Verbreitung besteht Grund, an der Zusammengehörigkeit dieser Wörter zu zweifeln.

Im Vergleich $\mathrm{zu}$ vielen anderen finnisch-ugrischen Sprachen erscheint der Wortschatz des Mari häufig intransparenter, weil dem Mari nahe verwandte Sprachen fehlen.

\section{Die Verweise auf das Tschuwaschische}

Der Verfasser und die Herausgeber haben sich dafür entschieden, mehreren Artikeln einen Kommentar anzufügen, der die Beziehung des marischen Wortschatzes 
zum Tschuwaschischen und zu ungefähr gleichbedeutenden, gleich aussehenden Wörtern im Tschuwaschischen behandelt. Tatsächlich ist eine etymologische Darstellung des marischen Wortschatzes sehr lückenhaft, wenn man den intensiven Einfluss der türkischen Sprachen, d. h. des Wolgabulgarischen, Tschuwaschischen und Tatarischen nicht berücksichtigt. Ein diesen Einfluss zusammenfassendes, aktuelles etymologisches Wörterbuch ist unbedingt $\mathrm{zu}$ begrüßen, denn die Veröffentlichung von Monografien zu diesem Thema liegt bereits weit zurück. Von den Mitwirkenden an dem hier zu besprechenden Buch hat Klára Agyagási selbst zahlreiche Beiträge zu dem Themenkreis publiziert.

Bei einem dem finnisch-ugrischen Wortschatz gewidmeten etymologischen Wörterbuch des Mari ist die Entscheidung, reichlich Platz für die Behandlung tschuwaschischer Parallelen zu opfern, widersprüchlich. Als Ausgangspunkt dient wiederholt der Gedanke, dass es sich um tschuwaschischen Einfluss handle, wenn ein Wort im Mari und im Tschuwaschischen die gleiche Grundbedeutung und $\mathrm{Be}$ deutungserweiterung aufweist oder in Komposita und Ableitungen auftritt. Diese Annahme ist von ihren Ausgangspunkten her problematisch, weil sie die Konvergenz, die innersprachliche Bedeutungserweiterung und die natürliche Polysemie der Wörter nicht berücksichtigt. Die durch Sprachkontakt verursachte Entwicklung wird $\mathrm{zu}$ einseitig auf den etymologischen Vergleich beschränkt. So hatte zum Beispiel der Kontakt mit den türkischen Sprachen zweifellos Einfluss auf die Entstehung der für das Mari charakteristischen Konverbkonstruktionen. Doch das bedeutet wohl nicht, dass jede Konverbkonstruktion, also sowohl das finite als auch das infinite Verb, wie kâlmen kolem, erfrieren (eig. ,erfrierend sterben')', dem Wörterbuch zufolge eine Lehnübersetzung aus der tschuwaschischen Verbverbindung šansa vil- id., auf lexikalischer Ebene eine Entsprechung im Tschuwaschischen hat, die die Entstehung der jeweiligen Konstruktion erklären würde. Beispielsweise gehören beide Bestandteile der marischen Verbverbindung kâlmen kolem zum alten Grundwortschatz finnisch-ugrischer Herkunft. Eine grammatikalische Kategorie kann entstehen und sich erweitern, ohne dass einzelne Wörter die Entwicklung steuern.

Eine ähnliche Hypothese vom Einfluss des Tschuwaschischen im Hintergrund der Polysemie und Bedeutungserweiterung wiederholt sich bei zahlreichen Wörtern, z.B. kijem, liegen', kit ,Hand', kučem, halten, fassen, besitzen', lektam , hinaus- 
gehen, weggehen', lijam ,sein, werden', moyâr ,Seite, Richtung', müškâr ,Bauch, Wanst', pengâ $\delta e$,hart, stark, haltbar, pâštem ,setzen, stellen, legen', poktem ,verfolgen, jagen, treiben', pot-koyolo ,Fleischklößchen', ter-, tor-, tör- etc. ,bei; zu, nach, mit', üঠem ,säen, pflanzen', waštareš ,gegenüber, entgegen'. In allen diesen Fällen wird die Erklärung dadurch ergänzt, dass die Bedeutungserweiterung auf tschuwaschischen Einfluss zurückgeführt wird oder beispielsweise die Verbverbindungen als Lehnübersetzungen dargestellt werden. Die Verwendung anthropomorpher Wörter, wie mel ,besticktes Bruststück des Hemdes; Brust ${ }^{\star}$ als Adposition zum Beispiel ist in den Sprachen der Welt weit verbreitet, sie ist einer der typischsten Grammatikalisierungspfade, die keine Erklärung durch Kontakte erfordern.

Die Beobachtungen sind zweifellos wichtig für die Ausweitung der Diskussion über den Einfluss der türkischen Sprachen auf das Mari, doch sie entsprechen im Grunde nicht dem Titel des Buches. Zudem wäre es angebrachter, sie in einem umfassenderen Kontext $\mathrm{zu}$ behandeln, in dem die Möglichkeit der Konvergenz, die Grammatikalisierung, die Erneuerung der Kategorien berücksichtigt, d.h. verschiedene Erklärungsalternativen beurteilt und verglichen werden. Ohne die Bedeutung des tschuwa- schischen Einflusses geringschätzen $\mathrm{zu}$ wollen, ist die Annahme, dass er sich entscheidend auf die Entwicklung Dutzender Wörter finnisch-ugrischer Herkunft im Mari ausgewirkt hätte, dennoch nicht begründet; anders verhält es sich mit der Veränderung der grammatikalischen Umgebung.

\section{Zum Schluss}

Im Vergleich $\mathrm{zu}$ vielen anderen finnisch-ugrischen Sprachen wirkt der Wortschatz des Mari häufig undurchsichtig, weil dem Mari eine als Vergleichsobjekt geeignete nahe verwandte Sprache fehlt. Die Dialekte des Mari wiederum stehen einander so nah, dass sie nur in Einzelfällen zusätzliches Licht auf die Geschichte der Wörter werfen. Das Etymologische Wörterbuch des Tscheremissischen (Mari) ist bestrebt, diesen Mangel zu beheben. Es ist die erste umfassende Gesamtdarstellung der finnisch-ugrischen oder als finnisch-ugrisch postulierten Wörter im Mari. Das posthume Werk von Gábor Bereczki zeigt, wie wichtig die Diskussion und die kritische Beurteilung wiederholt zitierter Etymologien sind.

Die ergänzenden Kommentare der Herausgeber zum Einfluss der türkischen Sprachen und besonders des Tschuwaschischen weisen den Weg in die Zukunft. Die ety- 
mologische Bestandsaufnahme des Wortschatzes des Mari ist so lange unvollständig, bis auch der aus dem Tatarischen und Tschuwaschischen entlehnte Wortschatz und die darin zum Ausdruck kommende Entwicklung der Sprache in das Gesamtbild einbezogen wird.

Besser geordnete und redigierte Wörterbücher schaffen hervorragende Voraussetzungen für die nachfolgenden Generationen, auf dem von Gábor Bereczki aufgezeigten Weg der etymologischen Erforschung des Mari voranzuschreiten. Die Perspektive, die die gemeinsame Frühgeschichte der finnischugrischen Sprachen eröffnet, kann unter vielen aktualisierten Aspekten auf das Mari angewandt werden. Auch im Hinblick auf die Kontakte zwischen den indogermanischen und den finnisch-ugri-

\section{Literatur}

Airio, Ante 2009: The Saami Loanwords in Finnish and Karelian. PhD thesis. University of Oulu.

- 2012 = LUObBal SÁmMOL SÁmMOL ÁNTE: On Finnic long vowels, Samoyed vowel sequences, and Proto-Uralic ${ }^{*} x$. - Tiina Hyytiäinen, Lotta Jalava, Janne Saarikivi \& Erika Sandman (hrsg.): Per Urales ad Orientem. Iter polyphonicum multilingue. Festskrift tillägnad Juha Janhunen på hans sextioårsdag den 12 februari 2012. Mémoires de la Société Finno-Ougrienne 264. Helsin- schen Sprachen ist eine vom Mari ausgehende Betrachtung fruchtbar.

Gábor Bereczki konnte sein Lebenswerk im Bereich des Mari zum Abschluss führen, doch das Erscheinen des gedruckten Buches durfte er nicht mehr erleben. Das Etymologische Wörterbuch des Tscheremissischen (Mari) ist ein wesentlicher Bestandteil des lebenslänglichen Engagements des Verfassers für die Erforschung der marischen Sprache und Kultur. Vervollständigt wird die Gesamtheit durch seine anderen Untersuchungen, wie die historische Morphologie des Mari A cseremisz nyelv történeti alaktana (2002). Sie bilden weiterhin das Fundament der Erforschung des Mari und sind ein wertvoller Führer in die sprachliche Frühgeschichte des Wolgagebiets.

Riho Grünthal ki: Finnisch-Ugrische Gesellschaft. 227-250.

BERECZKI, GÁBOR 1992-1994: Grundzüge der tscheremissischen Sprachgeschichte I-II. Studia Uralo-Altaica 34-35. Szeged.

EWU = Etymologisches Wörterbuch des Ungarischen. Hrsg. Loránd Benkő. Budapest: Akadémiai Kiadó. 19921997.

GRÜNTHAL, RIHO 2001: Indo-European echoes? Mordvin, Mari and Permic 'enter'. - Folia Uralica Debreceniensia 8: 189-198. 
- 2002: Prehistoric contacts between Mordvin and Indo-European. - Rogier Blokland \& Cornelius Hasselblatt (eds): Finno-Ugrians and Indo-Europeans: Linguistic and Literary Contacts. Proceedings of the Symposium at the University of Groningen, November 22-24, 2001. Studia Fenno-Ugrica Groningana 2. Shaker Publishing, Maastricht. 84-91.

JANHUNEN, JUHA 1981: Uralilaisen kantakielen sanastosta. - JSFOu 77: 219-274.

JokI, Aulis J. 1973: Uralier und Indogermanen. Mémoires de la Société Finno-Ougrienne 151. Helsinki: Finnisch-Ugrische Gesellschaft.

Koivulehto, Jorma 1999: Verba mutuata. Mémoires de la Société FinnoOugrienne 237. Helsinki: FinnischUgrische Gesellschaft.

Korhonen, Мiкко 1988: Uralilaisten kielten jälkitavujen vokaaliston historiaa. - Virittäjä 92: 8-25.

MSzFE = A magyar szókészlet finnugor elemei. Etimológiai szótár. Főszer- kesztő Lakó György, szerkesztő Rédei Károly. Budapest: Akadémiai kiadó 1967-1978.

Sammallahti, Pekka 1988: Historical phonology of the Uralic languages with special reference to Samoyed, Ugric and Permic. - Denis Sinor (ed.), The Uralic Languages - description, history and foreign influences. Leiden. 478-554.

SSA = ITKONEN, ERKKI - KulONEN, Ulla-Maija (eds) 1992-2000: Suomen sanojen alkuperä - etymologinen sanakirja. Helsinki: Suomalaisen Kirjallisuuden Seura.

TschWb = ARTO MOISIO UND SirkKa SAARINEn: Tscheremissisches Wörterbuch. Aufgezeichnet von Volmari Porkka, Arvid Genetz, Yrjö Wichmann, Martti Räsänen, T.E. Uotila und Erkki Itkonen. Lexica Societatis Fenno-Ugricae XXXII. Helsinki: Finnisch-Ugrische Gesellschaft 2008.

UEW = RÉDEI, KÁROLY 1988-1991: Uralisches Etymologisches Wörterbuch. Budapest: Akadémiai Kiadó. 